\title{
Prevalence of alpha-1-antitrypsin deficiency carriers in a population with and without colonic diverticula. A multicentre prospective case-control study: the ALADDIN study
}

\author{
S. J. Rottier ${ }^{1,2,3}$ (D) J. de Jonge ${ }^{2}$ - L. C. Dreuning ${ }^{2} \cdot$ J. van Pelt ${ }^{4}$ - A. A. W. van Geloven ${ }^{2}$ X. D. Y. Beele ${ }^{5}$ P. M. Huisman ${ }^{5} \cdot$ \\ W. Y. Deurholt ${ }^{6}$. C. A. Rottier ${ }^{1}$ - M. A. Boermeester ${ }^{3} \cdot$ W. H. Schreurs ${ }^{1} \cdot$ for the ALADDIN Collaborative Study Group
}

Accepted: 15 January 2019 / Published online: 15 February 2019

(C) The Author(s) 2019

\begin{abstract}
Purpose The underling pathophysiological mechanisms that cause the formation of colonic diverticula (diverticulosis) remain unclear. Connective tissue changes due to ageing that cause changes in collagen structure of the colonic wall is one theory. Alpha1 -antitrypsin (A1AT) is a protease inhibitor known to protect connective tissue in other organs. Associations between (carriers of) A1AT deficiency and the development of colonic diverticula will be the main focus of this study.

Methods A multicentre prospective case-controlled study. In total, 230 patients $\geq 60$ years with acute abdominal pain undergoing an abdominal computed tomography (CT) will be included. The research group consists of patients with diverticulosis and/or diverticulitis; controls are patients without diverticula ( 0 to $\leq 5$ diverticula). Genotype analysis for A1AT deficiency will be performed.

Rationale Hypothetically, connective tissue changes, in particular related to (carriers of) A1AT deficiency, can contribute to the development of diverticula and diverticulitis. We expect to find a higher prevalence of A1AT carriers in patients with diverticulosis compared to patients without diverticulosis. Having diverticulosis does not affect the general health of these individuals per se, when asymptomatic. Once an association is found, present findings can be the basis for a second study to assess the risk of developing acute diverticulitis and its disease course in carriers of A1AT deficiency. Because a large cohort is needed in the latter, we shall first perform a pilot study to investigate the likelihood of the primary hypothesis.
\end{abstract}

Trial registration Netherlands Trial register, NTR6251, NL55016.094.15

Keywords Diverticula $\cdot$ Diverticulosis $\cdot$ Diverticulitis $\cdot$ Alpha-1-antitrypsin $\cdot$ Connective tissue disease

S. J. Rottier

s.j.rottier@amc.nl; s.j.rottier@nwz.nl

1 Department of Surgery, Northwest Clinics, Wilhelminalaan 12, 1815 JD Alkmaar/Den Helder, Netherlands

2 Department of Surgery, Tergooi, Hilversum, Netherlands

3 Department of Surgery, Amsterdam UMC, Amsterdam, Netherlands

4 Department of Clinical laboratory, Northwest Clinics, Alkmaar/Den Helder, Netherlands

5 Department of Radiology, Tergooi, Hilversum, Netherlands

6 Department of Radiology, Northwest Clinics, Alkmaar/Den Helder, Netherlands

\section{Background}

The incidence of diverticulosis and its well-known complication diverticulitis is increasing worldwide and is imposing a growing burden on national healthcare systems [1]. Possible risk factors for developing diverticula are diet, obesity and alcohol consumption [2-4]. Possible risk factors for developing an episode of diverticulitis are medication such as corticosteroids, smoking and hereditary factors [5-7]. The underlying pathophysiological mechanism that causes the formation of colonic diverticula remains unclear. One theory is based on fibre deficiency, which was founded in 1971. This theory states that fibre deficiency results in decreased intestinal contents and a smaller size of intestinal lumen. Since the colonic muscles are contracting continuously in order to transmit and 
expel the stool, an increased intraluminal pressure is formed. This increased pressure leads to the formation of diverticula at the weakest anatomical locations in the wall. These predestined weak spots in the colonic wall are formed due to the entering of terminal branches of the colonic arteries and are referred to as vasa recta $[3,8]$. Other theories are based on the antimicrobial content of the colon, the microbiome theory [9].

Recently, a new hypothesis has emerged which can help us understand the aetiology of diverticulosis. This hypothesis is based on the fact that diverticulosis is an age-related disorder. Ageing eventually leads to the altering of colonic epithelia and it induces a declining colonic wall mechanical strength, which can be partly attributed to changes in collagen structure $[10$, 11]. One study has investigated how the collagen structure changes in colonic diverticulosis. Results indicated that colonic collagen from subjects affected by colonic diverticulosis had a higher number of cross-links than subjects with unaffected colonic tissue. This illustrates that the structural changes to tissue collagen affected by colonic diverticulosis have a greater impact than the changes that occur as part of the natural ageing process [12]. Not only a change in the number of cross-links but also a shift from stable collagen type 1 towards the less stable collagen type 3 and increased concentrations of tissuedegrading matrix metalloproteinases (MMPs) (for example MMP-1, MMP-2) and their inhibitor (TIMP-1) involved in connective tissue metabolism is observed in patients with (complicated) diverticular disease [13-15]. Consequently, colonic diverticulosis could be the result of an exaggerated and premature ageing process and intrinsic changes in collagen structure.

Alpha-1-antitrypsin (A1AT) deficiency is one of the most common hereditary disorders. It is assumed that there are at least 116 million carriers (such as PiMS and PiMZ) and 3.4 million deficiency allele combinations (PiSS, PiSZ and PiZZ) worldwide. It affects individuals from all racial subgroups worldwide [16]. A1 AT is a protease inhibitor of the proteolytic enzyme elastase that normally protects the connective tissue of the lungs when the leukocytes release elastase [17, 18]. Most patients with A1AT deficiency develop pulmonary emphysema and bronchitis [19]. One widely accepted theory about the pathophysiological mechanism that causes lung emphysema is the theory about an imbalance of protease and anti-protease, specifically elastase-anti elastase activities [20-22]. The interstitium of lung parenchyma consists mostly out of collagen type I and III, giving the alveolar wall its structural form [23]. Matrix metalloproteases (MMPs), which are proteolytic enzymes involved in tissue remodelling and repair associated with inflammation, are involved in maintaining the good structure of this wall $[22,24]$. Some of the MMPs involved in connective tissue metabolism are altered in patients with (complicated) diverticular disease. Those same MMPs (for example MMP-1 and MMP-2) are also altered in patients with lung emphysema [22, 25]. In some cases of true A1AT deficiency, the emphysema is severe and a lung transplantation is necessary to improve long-term survival [26]. In the field of lung transplant surgery, the correlation between A1AT deficiency and gastrointestinal complications has been highlighted. Gastrointestinal complications after lung transplantation variate from 3 to $51 \%$, and underlying mechanisms are poorly understood [27-29]. In A1AT deficiency, mortality in individuals with severe A1AT deficiency (type PiZZ) was increased due to respiratory and hepatic disease and pulmonary embolism, when compared to the age- and sex-matched Swedish population without this pathological allele for A1AT. Also, PiZZ individuals died more often due to complicated colonic diverticulitis [30]. These findings support our hypothesis that A1AT could be associated with the development of colonic diverticula.

Connective tissue diseases, in particular (carriers of) A1AT deficiency, may contribute to the development of diverticula and its complication diverticulitis. Expectations are to find a higher prevalence of A1AT carriers in patients with diverticulosis compared to patients without diverticulosis. The ALADDIN study aims to determine additional causes for developing colonic diverticulosis. An interesting hypothesis is that connective tissue diseases, in particular (carriers of) A1AT deficiency, contribute to the development of diverticulosis and its complication diverticulitis.

\section{Methods}

Study objective The aim of this study is to determine the prevalence of A1AT deficiency (carriers) in patients with and without colonic diverticula.

Study design This is a multi-centre prospective casecontrolled study. Patients who undergo an abdominal CT will be recruited at the emergency department (ED) of each participating medical centre. The $\mathrm{CT}$ will be reviewed by a radiologist who will report the diagnosis, the number of diverticula and the location of diverticula, and in case of diverticulitis, the Hinchey classification will be scored. Patients undergoing only other diagnostic methods, such as ultrasound or colonoscopy, will be excluded as will be unable to give this additional information and were therefore excluded. Blood samples will be collected in order to determine the concentration of A1AT in serum by immunoassay and genotype analysis will be performed. Genotyping will reveal whether an individual has a normal variant (PiMM), is carrier of A1AT deficiency (for example PiMS, PiMZ or PiMF) or has A1AT deficiency (PiSS, PiSZ and PiZZ). Genotype analysis uses allelespecific amplification that allows variables to be identified. Although the optimum formula for A1AT deficiency testing 
has not been formulated, serum level measurement combined with genotype analysis is often used in clinical setting.

Outcome measures Outcome measures will be (a) prevalence of A1AT deficiency or carriers in patients with and without colonic diverticula, (b) the number of episodes of diverticulitis in patients with and without (carrier alleles for) A1AT deficiency and (c) the number of diverticulitis-related hospital admissions in patients with and without (carrier alleles for) A1AT deficiency. Evaluation of known risk factors for developing diverticulosis, e.g. genetic factors, environmental factors and epidemiological factors, will be collected using a questionnaire and medical records.

Study population All patients $\geq 60$ years, presenting at the ED with acute abdominal pain and undergoing an abdominal CT, will be included consecutively. Acute abdominal pain is defined as non-traumatic abdominal pain with symptom duration of more than $2 \mathrm{~h}$ and less than 5 days [31]. Patients are eligible for the research group when the abdominal CT reveals diverticula. Patients without diverticula defined as 0 to $\leq 5$ diverticula on abdominal CT will be included in the control group (Figs. 1 and 2). All patients eligible for this study will be screened by the attending emergency physician for mentally competence; patients have to be able to give informed consent and fill out a questionnaire. The questionnaire aims to identify known risk factors for developing diverticula or acute diverticulitis, such as diet focusing on fibre rich or poor, BMI, smoking and pack years, use of medication such as NSAIDs or corticosteroids, ethnicity, family history of diverticulitis, previous episodes of diverticulitis and previously undergone treatment for acute episodes of diverticulitis.
Ethical considerations The study is conducted in accordance with the principles of the Declaration of Fortaleza and 'good clinical practice' guidelines. The protocol (version 4, amendment 3, May 2, 2018) has been approved by the Medical Research Ethics committees of VU University Medical Center (MREcVUmc). Consent was also obtained from the participating centres. Patients will be counselled and written informed consent will be obtained from all patients if inclusion criteria have been met.

Laboratory analysis An Amplicon sequencing (Ampliseq) panel with coding sequences (CDS) was designed for three known A1AT deficiency-causing genes (SERPINA1, SERPINA3 and ELA2). This panel will be used to create amplicons out of the isolated deoxyribonucleic acid (DNA). Ion Chef will be used to create a nextgeneration sequencing (NGS) library. These libraries will be pooled on an Ion chip and sequenced to an ION S5 sequencer (Thermofisher). Variants will be called up on using an Ion reporter server. Then, reported variants will be compared to known variants in the Human Gene Mutation Database (HGMD).

Statistical analysis Nominal and ordinal variables will be described as numbers with proportions. Continuous variables will be described as means with standard deviations (SD) or medians with interquartile ranges (IQR). Between-group (case vs controls) differences with respect to primary and secondary study parameters will be analysed using the chi-square test or the Fisher exact test in case of nominal and ordinal variables. Differences with respect to continuous variables will be analysed using the independent samples $t$ test or the MannWhitney $U$ test depending on the distribution. The strength of
Fig. 1 The ALADDIN study flowchart, study design

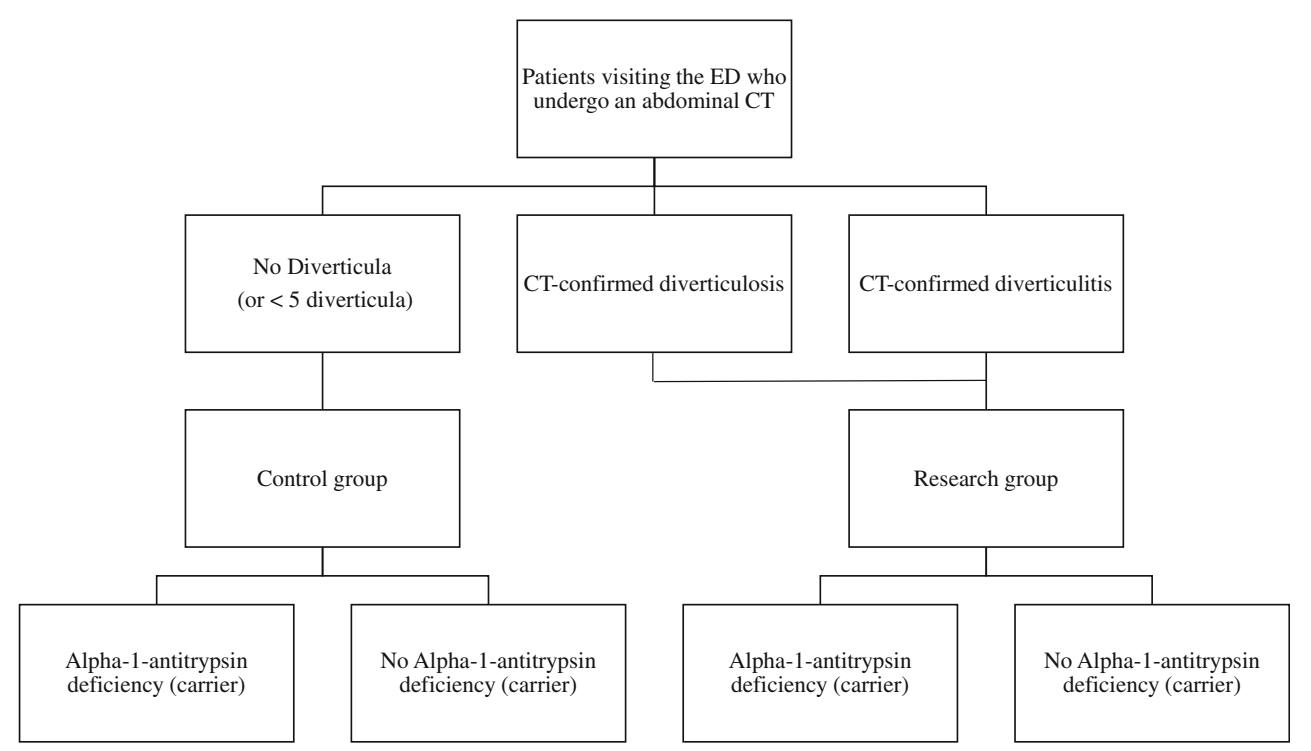




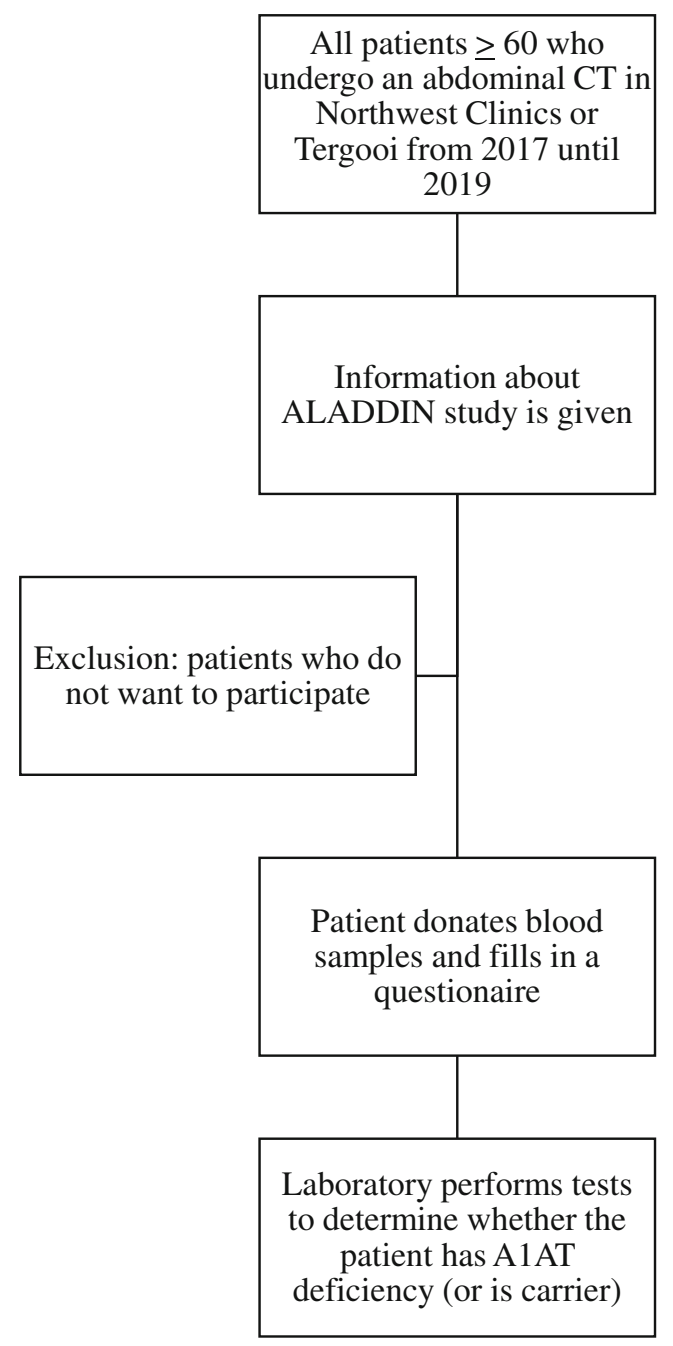

Fig. 2 The ALADDIN study flowchart, composing study population

the association between exposure of alpha-1-antitrypsin pathology and the occurrence of diverticulosis will be analysed by means of logistic regression analysis and expressed as an odds ratio (OR) with a $95 \%$ confidence interval $(95 \% \mathrm{CI})$. Multivariable logistic regression analysis will be used to correct this association for confounding effects. All secondary study parameters (except for alpha-1-antitrypsin concentration) are potential confounders and are classified as such if they change the regression coefficient of the primary variable by more than $10 \%$ when added to the regression analysis. All other study parameters will be analysed in patients with diverticula and compared between patients with and without the alpha-1-antitrypsin deficiency (carriers). Differences with respect to continuous variables will be analysed using the independent samples $t$ test or the Mann-Whitney $U$ test depending on the distribution. Differences with regard to nominal or ordinal variables will be analysed using the chi-square test.

The STROBE guidelines for reporting will be followed [32]. All analyses will be performed using SPSS, version 24.0 (SPSS Inc., Chicago, IL, USA).
Sample size calculation It is assumed that there are at least 116 million carriers (PiMS and PiMZ) and 3.4 million deficiency allele combinations (PiSS, PiSZ and PiZZ) worldwide. The prevalence of A1AT deficiency carriers in Europe was calculated to be approximately $8 \%$ [16]. No previous information on the prevalence of the A1AT deficiency (carriers) in patients with or without diverticula exists. Therefore, we can only speculate on the possible difference in prevalence between these two groups. Assuming a $10 \%$ difference in prevalence and using a significance level of $5 \%$ and a power of $80 \%, 115$ patients per group would be necessary. Since literature indicates that prevalence of diverticulosis is around $50 \%$, inclusion of cases and controls is expected to progress equally $[33,34]$.

Data collection and monitoring Data will be collected from the medical records of the patients. Additional information will be gathered using a questionnaire. Patient data will be coded using a unique study code and stored electronically on an eCRF using the data management software Castor EDC.

\section{Rationale for the design}

The aim of this study was to assess possible associations between CTD and the development of diverticula, with special interest in (carriers of) A1AT deficiency, and also the association between the amount and severity of episodes of acute diverticulitis in patients with (carrier alleles for) A1AT deficiency and those without. Therefore, we need patients with acute abdominal pain in whom an abdominal CT is performed, since this provides information regarding the presence of diverticula, the number and location of the diverticula and - if applicable - information about the severity of the diverticulitis episode. With the use of abdominal CT in the acute stage of diverticulitis, it is possible to classify these patients according to the Hinchey classification. The consequence of this design, however, is that the results of this study will only be applicable to patients with acute abdominal pain. Nevertheless, the use of other diagnostic methods to select patients will be accompanied with other disadvantages. An alternative would be to include patients from the national screening programme undergoing a colonoscopy, but this will produce no data for the secondary outcome measures, as colonoscopy is not performed during an acute episode of diverticulitis. Moreover, the indication for a screening colonoscopy in the Netherlands is a positive immunochemical faecal occult blood test (iFOBT); therefore, the population selected through this programme will not be completely asymptomatic. 


\section{Discussion}

Our hypothesis is that connective tissue diseases, in particular A1AT deficiency or being carrier of this deficiency, contribute to the development of diverticula. Because the prevalence of A1AT deficiency is low, approximately $0.24 \%$ in Europe, the focus will be on the prevalence of A1AT deficiency carriers, having a prevalence of $8 \%$ [16]. Expectations are to find a higher prevalence of A1AT deficiency carriers in patients with diverticulosis (cases) compared to patients without diverticulosis (controls). We estimate the prevalence to be around $13 \%$ in the cases and less than $3 \%$ in the control group. If the association between A1AT deficiency and diverticula formation is confirmed, this finding is just a start. Having diverticulosis does not affect the general health of these individuals per se, when asymptomatic. However, this study can be the basis for a second study to see if patients who are carrier of A1AT deficiency also have a higher risk of developing acute diverticulitis, and if so, whether disease course is more severely. Because a large cohort is needed in the latter, we shall first perform a pilot study to investigate the likelihood of the primary hypothesis. We realise that the results of this study will only be applicable on our selected population, being patients with acute abdominal pain.

Because diverticulitis is a multifactorial disease and probably a result of complex interactions, it may be hard to prove that deviations of the A1AT protein play a role in the aetiology of diverticulitis [9]. Little or no information is available in the current literature. The focus of this study is mainly on one connective tissue disease, A1AT deficiency. However, the final goal is to investigate multiple connective tissue diseases. Because a large cohort is needed in the latter, the decision was made to first perform a pilot study to investigate the likelihood of the hypothesis.

When our theory will be confirmed by performing this study, this could not only affect treatment standards for lung transplant patients, but also generate a different theoretical context for patients in general diagnosed with acute diverticulitis. It is a starting point for further investigations in a large cohort of diverticulitis patients, once an association is confirmed in present study. When patients who are carrier for A1AT deficiency have significant more episodes of diverticulitis and these episodes progress more severely compared to the patients with a normal type of A1AT, this information can help a physician to treat a patient. Furthermore, this information can help to decide if in a selected group (being patients with A1AT deformations), hospital admission is necessary or antibiotic treatment should be given. It could also help in the decision whether early surgical intervention is justified or if a more conservative treatment (especially in patients with normal
A1AT) is accepted. Better understanding of the association between A1AT deficiency and diverticula development could contribute to changes in the treatment of diverticulitis. It could also be the foundation of a screening programme for patients who have to undergo a lung transplantation because of severe A1AT deficiency.

Acknowledgements W.M. van Vuuren, Pre-analysis Clinical Chemical Hematological Laboratory Tergooi, W.K.C; Koot-Peet, Pre-analysis Clinical Chemical Hematological Laboratory Tergooi; L. Hoogendoorn, Clinical Chemical laboratory NWZ; I. van der Hulst, Clinical Chemical Laboratory NWZ; G. van Mierlo, Department of Immunopathology, Sanquin Blood Supply, Division Research and Landsteiner Laboratory of the Academic Medical Center, University of Amsterdam; K. van Leeuwen, Department of Blood Cell Research, Sanquin Blood Supply, Division Research and Landsteiner Laboratory of the Academic Medical Center, University of Amsterdam; M. de Boer, Department of Blood Cell Research, Sanquin Blood Supply, Division Research and Landsteiner Laboratory of the Academic Medical Center, University of Amsterdam

Funding The ALADDIN study received no specific grant from any funding agency in the public of commercial sectors. We did receive local funding from the participating local hospitals (Northwest clinics, location Alkmaar, the Netherlands and Tergooi, Hilversum, the Netherlands) to pay for the genotype analysis and salary of the $\mathrm{PhD}$ student.

\section{Compliance with ethical standards}

Trial status The protocol (version 4, amendment 3, May 2, 2018) has been approved by the Medical Research Ethics committees of VU University Medical Center (MREcVUmc). We included just over 200 patients in the ALADDIN study at the time of submission of the protocol to International Journal of Colorectal Disease (November 2018). The first patient was included in the Northwest Clinics in Alkmaar, Netherlands.

Conflict of interest The authors declare that they have no conflict of interest.

Research involving human participants and/or animals All procedures performed in studies involving human participants were in accordance with the ethical standards of the institutional and/or national research committee and with the 1964 Helsinki declaration and its later amendments or comparable ethical standards. The protocol (version 4, amendment 3, May 2, 2018) has been approved by the Medical Research Ethics committees of VU University medical center (MREcVUmc), number 2016.900 (A2018.242).

Informed consent Informed consent was obtained from all individual participants included in the study.

Open Access This article is distributed under the terms of the Creative Commons Attribution 4.0 International License (http:// creativecommons.org/licenses/by/4.0/), which permits unrestricted use, distribution, and reproduction in any medium, provided you give appropriate credit to the original author(s) and the source, provide a link to the Creative Commons license, and indicate if changes were made.

Publisher's note Springer Nature remains neutral with regard to jurisdictional claims in published maps and institutional affiliations. 


\section{References}

1. Tursi A, Papa A, Danese S (2015) Review article: the pathophysiology and medical management of diverticulosis and diverticular disease of the colon. Aliment Pharmacol Ther 42(6):664-684

2. Sharara AI, El-Halabi MM, Mansour NM, Malli A, Ghaith OA, Hashash JG et al (2013) Alcohol consumption is a risk factor for colonic diverticulosis. J Clin Gastroenterol 47(5):420-425

3. Burkitt DP, Walker AR, Painter NS (1972) Effect of dietary fibre on stools and the transit-times, and its role in the causation of disease. Lancet 2(7792):1408-1412

4. Comstock SS, Lewis MM, Pathak DR, Hortos K, Kovan B, Fenton JI (2014) Cross-sectional analysis of obesity and serum analytes in males identifies sRAGE as a novel biomarker inversely associated with diverticulosis. PLoS One 9(4):e95232

5. Granlund J, Svensson T, Olen O, Hjern F, Pedersen NL, Magnusson PK et al (2012) The genetic influence on diverticular disease-a twin study. Aliment Pharmacol Ther 35(9):1103-1107

6. Hjern F, Mahmood MW, Abraham-Nordling M, Wolk A, Hakansson N (2015) Cohort study of corticosteroid use and risk of hospital admission for diverticular disease. Br J Surg 102(1): 119-124

7. Hjern F, Wolk A, Hakansson N (2011) Smoking and the risk of diverticular disease in women. Br J Surg 98(7):997-1002

8. Painter NS (1968) Diverticular disease of the colon. Br Med J 3(5616):475-479

9. Daniels L, Philipszoon LE, Boermeester MA (2014) A hypothesis: important role for gut microbiota in the etiopathogenesis of diverticular disease. Dis Colon Rectum 57(4):539-543

10. Szojda MM, Cuesta MA, Mulder CM, Felt-Bersma RJ (2007) Review article: management of diverticulitis. Aliment Pharmacol Ther 26(Suppl 2):67-76

11. Watters DA, Smith AN, Eastwood MA, Anderson KC, Elton RA, Mugerwa JW (1985) Mechanical properties of the colon: comparison of the features of the African and European colon in vitro. Gut 26(4):384-392

12. Wess L, Eastwood MA, Wess TJ, Busuttil A, Miller A (1995) Cross linking of collagen is increased in colonic diverticulosis. Gut 37(1): 91-94

13. Bode MK, Karttunen TJ, Makela J, Risteli L, Risteli J (2000) Type I and III collagens in human colon cancer and diverticulosis. Scand $\mathrm{J}$ Gastroenterol 35(7):747-752

14. Rosemar A, Ivarsson ML, Borjesson L, Holmdahl L (2007) Increased concentration of tissue-degrading matrix metalloproteinases and their inhibitor in complicated diverticular disease. Scand J Gastroenterol 42(2):215-220

15. Wedel T, Barrenschee M, Lange C, Cossais F, Bottner M (2015) Morphologic basis for developing diverticular disease, diverticulitis, and diverticular bleeding. Viszeralmedizin 31(2):76-82

16. de Serres FJ (2002) Worldwide racial and ethnic distribution of alpha1-antitrypsin deficiency: summary of an analysis of published genetic epidemiologic surveys. Chest 122(5):1818-1829

17. Carrell RW, Lomas DA (2002) Alpha1-antitrypsin deficiency-a model for conformational diseases. N Engl J Med 346(1):45-53
18. Stoller JK, Aboussouan LS (2012) A review of alpha1-antitrypsin deficiency. Am J Respir Crit Care Med 185(3):246-259

19. Eriksson S (1965) Studies in alpha 1-antitrypsin deficiency. Acta Med Scand Suppl 432:1-85

20. Stockley RA (1999) Neutrophils and protease/antiprotease imbalance. Am J Respir Crit Care Med 160(5 Pt 2):S49-S52

21. Evans MD, Pryor WA (1994) Cigarette smoking, emphysema, and damage to alpha 1-proteinase inhibitor. Am J Phys 266(6 Pt 1): L593-L611

22. Ohnishi K, Takagi M, Kurokawa Y, Satomi S, Konttinen YT (1998) Matrix metalloproteinase-mediated extracellular matrix protein degradation in human pulmonary emphysema. Lab Investig 78(9): 1077-1087

23. Suki B, Bates JH (2008) Extracellular matrix mechanics in lung parenchymal diseases. Respir Physiol Neurobiol 163(1-3):33-43

24. Nagase H, Woessner JF Jr (1999) Matrix metalloproteinases. J Biol Chem 274(31):21491-21494

25. McAloon CJ, Wood AM, Gough SC, Stockley RA (2009) Matrix metalloprotease polymorphisms are associated with gas transfer in alpha 1 antitrypsin deficiency. Ther Adv Respir Dis 3(1):23-30

26. Tanash HA, Riise GC, Hansson L, Nilsson PM, Piitulainen E (2011) Survival benefit of lung transplantation in individuals with severe alpha1-anti-trypsin deficiency (PiZZ) and emphysema. J Heart Lung Transplant 30(12):1342-1347

27. Bredahl P, Zemtsovski M, Perch M, Pedersen DL, Rasmussen A, Steinbruchel D et al (2014) Early laparotomy after lung transplantation: increased incidence for patients with alpha1-anti-trypsin deficiency. J Heart Lung Transplant 33(7):727-733

28. Hoekstra HJH, K.; de Boer, W.J.; Rottier, K.; van der Bij, W. Gastrointestinal complications in lung transplant survivors that require surgical intervention. Britisch J Surg 2001;88:433-438

29. Leonardi MJ, Jamil KG, Hiscox B, Ross D, Hiatt JR (2010) Abdominal surgery after lung transplantation. Am Surg 76(10): $1130-1134$

30. Tanash HA, Ekstrom M, Wagner P, Piitulainen E (2016) Causespecific mortality in individuals with severe alpha 1-antitrypsin deficiency in comparison with the general population in Sweden. Int J Chron Obstruct Pulmon Dis 11:1663-1669

31. Lameris W, van Randen A, van Es HW, van Heesewijk JP, van Ramshorst B, Bouma WH et al (2009) Imaging strategies for detection of urgent conditions in patients with acute abdominal pain: diagnostic accuracy study. BMJ 338:b2431

32. von Elm E, Altman DG, Egger M, Pocock SJ, Gotzsche PC, Vandenbroucke JP et al (2007) The Strengthening the Reporting of Observational Studies in Epidemiology (STROBE) statement: guidelines for reporting observational studies. Lancet 370(9596): 1453-1457

33. De Cecco CN, Ciolina M, Annibale B, Rengo M, Bellini D, Muscogiuri G et al (2016) Prevalence and distribution of colonic diverticula assessed with CT colonography (CTC). Eur Radiol 26(3):639-645

34. Golder M, Ster IC, Babu P, Sharma A, Bayat M, Farah A (2011) Demographic determinants of risk, colon distribution and density scores of diverticular disease. World J Gastroenterol 17(8):10091017 